\title{
Les versions en prose du Purgatoire de saint Patrice en ancien français. Édition critique, introduction et notes par Martina Di Febo
}

\section{Maria Colombo Timelli}

\section{(C) OpenEdition}

\section{Journals}

\section{Édition électronique}

URL : http://journals.openedition.org/studifrancesi/1077

DOI : 10.4000/studifrancesi. 1077

ISSN : 2421-5856

Éditeur

Rosenberg \& Sellier

\section{Édition imprimée}

Date de publication : 1 novembre 2014

Pagination : $567-568$

ISSN : 0039-2944

\section{Référence électronique}

Maria Colombo Timelli, « Les versions en prose du Purgatoire de saint Patrice en ancien français. Édition critique, introduction et notes par Martina Di Febo », Studi Francesi [En ligne], 174 (LVIII | III) | 2014, mis en ligne le 01 novembre 2014, consulté le 18 septembre 2020. URL : http://journals.openedition.org/ studifrancesi/1077; DOI : https://doi.org/10.4000/studifrancesi.1077

Ce document a été généré automatiquement le 18 septembre 2020.

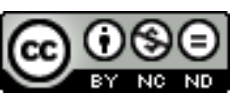

Studi Francesi è distribuita con Licenza Creative Commons Attribuzione - Non commerciale - Non opere derivate 4.0 Internazionale. 


\title{
Les versions en prose du Purgatoire de saint Patrice en ancien français. Édition critique, introduction et notes par Martina Di Febo
}

\author{
Maria Colombo Timelli
}

\section{RÉFÉRENCE}

Les versions en prose du Purgatoire de saint Patrice en ancien français. Édition critique, introduction et notes par Martina DI FEBO, Paris, Honoré Champion, 2013 («CFMA», 172), pp. 294.

1 Le Tractatus de purgatorio sancti Patricii, texte anonyme rédigé vers 1173-1185, a connu une fortune immense au Moyen Âge et au-delà, avec des traductions dans toutes les langues romanes et germaniques: pour ce qui concerne le français, on ne dénombre pas moins de six traductions en vers et quatre en prose, du XII au XVI ${ }^{e}$ siècle. Martina Di Febo offre ici l'édition de la version en prose la plus ancienne ( $a$, début du XIII ${ }^{\mathrm{e}}$ siècle) et d'un remaniement de celle-ci ( $a^{1}, \mathrm{XIV}^{\mathrm{e}}$ siècle), qui dérivent de la rédaction alpha du traité latin; elle ajoute une version plus tardive (e, également du xIv siècle), qui témoigne d'une contamination probable entre les versions latines alpha et bêta. L'Introduction permet de faire le point sur une situation textuelle complexe, en offrant d'abord les informations essentielles sur le texte latin (schéma du contenu des rédactions alpha et bêta, p. 16-17), puis l'analyse des versions françaises en prose ( $a / a^{1}, b, e, f ;$ p. 18-36).

Les éditions elles-mêmes constituent trois sections indépendantes. La version $a$ est transmise par 33 manuscrits, que M. Di Febo décrit p. 37-59 (on signalera entre parenthèses que le Marquis de Paulmy n'a pas «hérité [...] la bibliothèque du Duc de La vallière», comme il est dit p. 55 à propos du ms.Arsenal 3145); une collation très minutieuse et approfondie (p. 59-81) lui a permis d'aboutir à un stemma (p. 81) et de 
choisir son texte de base ( $P 4$, voir p. 60). L'analyse linguistique (p. 82-87) et la «présentation du texte» (de fait, il s'agit du «traitement du t.», p. 87-90) complètent la section liminaire. Cette première version de la Vie monsigneur seint Patrice - tel est le titre qui se lit dans l'incipit - (p. 91-112) peut être divisée, sur la base des lettrines du ms, en 28 paragraphes, qui ne correspondent cependant ni à une subdivision logique du texte ni aux étapes de l'itinéraire ultra-mondain suivi par le protagoniste. Quelques choix dans la transcription nous laissent perplexe: ainsi, par exemple, pour ce qui concerne les lectures $n i / n ' i$ (nus ni peut entrer 1,74; que il ni entrast 2,13; il ni remest neïs une estincele 6,10-11; n'onques riens ni vit 6,19; et ainsi de suite) ou si / s'i (home qui si fiast 3,28). La distinction $i / j$ n'est pas toujours respectée: pourquoi Iesucrist 7,30, ieterent (verbe jeter) 9,25, ou encore ienouz 10,19 , iustes 23,17 , ietéz 27,23 ? Plus grave: l'odeur qui se dégage d'un des «enfers» visités par la protagoniste est telle que «se toz li mondes fust espices (lire: espicés) ne li semblast il mie qu'il i eüst plus grant odeur...» 17,6; desoremes 23,18, devrait aussi porter un accent; au contraire, on lira «ceste viande est apareillie (et non pas apareillié: le texte présente de nombreux traits picards: $c f . p .82$ ) sanz fin a ceus qui de ci monteront el ciel» 23,11. L'apparat critique (p. 113-116) enregistre les variantes importantes des autres manuscrits et constitue par là une véritable mine d'informations, alors que les «notes critiques» (p. 137-148) offrent des commentaires complémentaires.

3 L'édition de $a^{1}$ suit le même schéma; il s'agit, comme on l'a dit, d'une rédaction abrégée de $a$ transmise par un seul manuscrit, du $\mathrm{xv}^{\mathrm{e}}$ siècle. Parmi ses caractéristiques, rappelons au moins que la division en chapitres est ici marquée par des titres, ce qui donnait aux lecteurs de l'époque de commodes points de repère. Sans entrer dans les détails, remarquons - parmi beaucoup d'autres - quelques leçons sans doute à revoir, ou tout au moins à discuter: la graphie Islande pour Irlande $(1,2$ et 1,19$)$ aurait mérité un commentaire, en note ou dans l'introduction linguistique; par cesse fosse $(2,30)$ doit sans doute se lire par ceste f:; Et lors leur donnerent $(3,41)$ doit probablement être corrigé en lui d., s'agissant des messagers qui donnent leur benichon à Owen; dans les dyables [...] qu'ilz les batoient $(7,13)$, lire quilz; la leçon Mais le largue vist il bien car par la entra et yssit ne me paraît pas claire (7,15: le substantif n'est d'ailleurs pas enregistré dans le glossaire): elle s'éclaircit en comparant ce passage avec le texte de a: mes la largesce vit il bien, 8,14).

Quant à la version $e$, il s'agit d'une nouvelle traduction remontant au milieu du XIv ${ }^{e}$ siècle, transmise par trois manuscrits, dont deux du $\mathrm{Xv}^{\mathrm{e}}$ et un du $\mathrm{xvI}^{\mathrm{e}}$. Dans ce cas encore, je me limiterai à signaler des doutes de lecture: icelle folle à lire selon toute probabilité fosse (LVIII, 34); seust bien tenuz, sans doute feust (LXII, 32); il sera moult que sage, plutôt il fera (LXXIII, 25). Deux intégrations me paraissent franchement superflues: «Et tant plus aproucha, de tant plus >cler< les oït» (LXIII, 12); «Et estoient les differences sy plaisans que >c'estoit< merveilles» (LXXI, 53); en l'absence de notes à ces deux passages, il est cependant difficile de comprendre ce qui les aurait motivées.

5 Le glossaire (p. 249-262) et l'index des noms propres (p. 263-264) couvrent les trois éditions. La bibliographie, raisonnée et très complète, occupe quelque 25 pages en petits caractères (p. 265-291).

6 Le véritable problème de cette édition est cependant représenté par la langue française utilisée par l'éditrice: sans trop insister, notons que les fautes sont si diverses et nombreuses (italianismes, erreurs dans la morphosyntaxe, dans le lexique) qu'elles en arrivent à entraver la compréhension de certains passages. Les sigles utilisés pour désigner les différentes versions du texte, latines et françaises, posent aussi des 
problèmes: ainsi dans la table p. 33, le sigle «b» doit probablement être lu «e», puisque c'est de cette version que l'on discute; dans les titres des trois rédactions éditées, p. 37, 149 , 195, les sigles «a», «a ${ }^{1} »$ et «e» sont majuscules, ce qui crée une confusion regrettable avec les sigles désignant les manuscrits; de même, «B» p. 28 doit être lu «b», s'agissant de la version et non pas d'un ms, ainsi que «E»p. 220, à lire «e» pour la même raison.

7 Sans aucun doute, une relecture même rapide de la part du Comité de Publication des «CFMA» aurait évité la parution d'une édition importante dont la valeur risque de passer au second plan par rapport à une présentation des plus fautives. 